\title{
Effective Utilization and Appropriate Selection of Genetically Engineered Mouse Models for Translational Integration of Mouse and Human Trials
}

\author{
Cory Abate-Shen ${ }^{1,3}$ and Pier Paolo Pandolfi ${ }^{2,3}$ \\ ${ }^{1}$ Departments of Urology and of Pathology and Cell Biology, Herbert Irving Comprehensive Cancer Center, \\ Columbia University Medical Center, New York, New York 10032; ${ }^{2}$ Cancer Genetics Program, Beth Israel \\ Deaconess Cancer Center, Department of Medicine and Pathology, Beth Israel Deaconess Medical Center, Harvard \\ Medical School, Boston, Massachusetts 02215
}

The landscape of cancer research and therapy has radically changed over the past decades in at least two major respects: Our ability to model cancer in the mouse has risen to an unprecedented level of accuracy at the same time that novel cancer drugs have been developed in record numbers. This has led to an explosion in genetically engineered mouse model (GEMM) research, as GEMMs can potentially be used to test and optimize drugs in a variety of ways: preclinically (before testing in human patients), coclinically (in parallel with human testing), and postclinically (to optimize standard of care therapy). Thus the potential applications of faithful GEMMs of cancer have expanded from analysis of causal relationships between genetic aberrancies and tumorigenesis in preclinical efforts to a more comprehensive and systematic utilization of GEMMs for drug testing and clinical trial optimization. As GEMM research has grown, however, few standard protocols have been put in place regarding GEMM trials done in parallel with human trials (the "coclinical" approach), or in situations in which the available cohort of human patients is too small for valid statistical analysis. The success of such efforts will require an increased attention to the rigor with which mouse and human clinical efforts are designed, executed, and integrated.

A growing appreciation of the overwhelming genetic complexity underlying human cancer underpins contemporary cancer research and medical oncology. By now it is fully recognized that cancer develops in many different genetic subtypes and appreciated that this diversity impacts how these tumors respond to or resist various forms of therapy. At the same time, it is widely understood that an unprecedented therapeutic opportunity has arisen, in that we have a large number of new experimental drugs available for rapid testing in many cancer subtypes. This opportunity in turn creates new challenges in the appropriate design of clinical trials. One major hurdle is represented by the fact that if cancer occurs in many subtypes, and there are many drugs to be tested (both singly and combinatorially), the number of patients with each subtype who are available for clinical trials will become ratelimiting, whereas the process of testing multiple or combinatorial therapies may be prolonged.

In overcoming these limitations, accurate GEMMs of specific cancer subtypes have proven instrumental; indeed, they have already guided and optimized the treatment of several forms of human

\footnotetext{
${ }^{3}$ Correspondence: cabateshen@columbia.edu; ppandolf@bidmc.harvard.edu

(C) 2013 Cold Spring Harbor Laboratory Press

Cite this introduction as Cold Spring Harb Protoc; 2013; doi:10.1101/pdb.top078774
} 
cancer. A paradigmatic example is represented by acute promyelocytic leukemia (APL), a distinct form of leukemia, which was discovered to be genetically heterogeneous, with six different subtypes variably responsive to therapy (Nardella et al. 2011). Patient accrual in clinical trials for these six subtypes of APL would have been a daunting hurdle. GEMMs of each APL subtype, however, overcame the problem and proved invaluable to optimizing targeted combinatorial treatments in a preclinical setting; successful combinatorial treatments subsequently proved curative in human APL, just as they had in GEMM "surrogate patients" (Nardella et al. 2011). In recent years, the use of GEMMs has further expanded to meet the needs and complexity of modern oncology. Here we will discuss guiding principles and appropriate general standard operating procedures for the use of GEMMs in guiding clinical efforts in oncology.

\section{OPTIMIZING THE USE OF MODELS FOR PRECLINICAL, COCLINICAL, AND POSTCLINICAL TRIALS}

The most established use of GEMMs of cancer and other diseases has been represented, historically, by the "preclinical" testing of a given compound, in what are commonly referred to as "proof-ofprinciple" experiments designed to prove or disprove the efficacy of a given treatment modality (Box 1). The preclinical use of GEMMs has been of great medical benefit, as it can largely anticipate and guide the subsequent clinical testing of a given drug or drug combination. This classic approach has led to many therapeutic successes (e.g., the above-mentioned APL paradigm reviewed in Nardella et al. 2011).

In considering how to best use a GEMM "preclinically" and maximize the information obtained from such an effort, it is of paramount importance to replicate the modality by which the drug will eventually be used in the human clinical setting. As an example, if the experimental drug is to be a second-line treatment modality, it is optimal to design the "proof-of-principle" preclinical GEMM effort in strict alignment with the sequence of events that will take place in the human trial: that is, the first-line standard of care should precede the second-line "experimental" drug in the GEMMs under study just as it will in human patients, because of the concern that a pretreated model/tumor may be biologically and genetically different from an untreated model/tumor.

Although the "preclinical" dimension should be exploited whenever possible and never abandoned, two additional mode of use for GEMMs (see Box 1) can be of tremendous value now that many faithful GEMMs for a given form of cancer are available, and many novel drugs are being evaluated in clinical trials. The fact that there are numerous new experimental drugs in the clinic opens the possibility of aligning preclinical efforts in GEMMs with clinical trail efforts in humans. The essence of this approach, which we refer to as "coclinical" (see Box 1 and Nardella et al. 2011 for a more detailed description of the "coclinical" approach) rests in the rigorous alignment and synchronization of the clinical trial in human patients with the preclinical trial in GEMMs, and the enrollment of as many GEMMs representative of the genetic diversity of a given tumor type (e.g., multiple, genetically diverse GEMMs of prostate or lung cancer) in which the human trial is performed. Once again, the aligned execution of these "coclinical" trials in comparable ways is of critical impor-

\section{BOX 1. UTILIZATION MODALITIES OF GEMMs}

"Preclinical": Before Phase I trials Example: Test consequences of androgen inhibitors in prostate cancer GEMMs

"Coclinical": Concomitantly with Phase I/Phase I-II trials Example: Evaluate androgen inhibitors in prostate cancer GEMMs in parallel with clinical trials

"Postclinical": To optimize standard of care (SOD) treatment modalities Example: Optimize androgen deprivation therapy in prostate cancer GEMMs 
tance in respect to dosing, scheduling, and route of administration. Similarly, the data accrued should be integrated for comparison as we discuss below.

Another application of GEMMs, which will yield critical insights into how cancer treatment is optimized for years to come, is their use in a "postclinical" setting. In these protocols, GEMMs representative of the genetic diversity of a given tumor type are enrolled in standard-of-care (SOC) trials in an attempt to develop an accurate genetic stratification for therapies currently prescribed for human patients (e.g., androgen deprivation in prostate cancer or radiation therapy in brain tumors) (see Box 1). Alternatively, they can be enrolled in studies of agents that failed in clinical trials to better understand the outcome and how they might better be used in future efforts.

Important goals of pre-, co-, and postclinical trials with GEMMs include the accurate genetic stratification of response as observed in an accelerated manner in vivo, and the similarly rapid identification of novel biomarkers and mechanisms of resistance. Yet the objectives that a specific clinical use of GEMMs can help achieve are multiple and diverse (Box 2).

\section{SELECTING APPROPRIATE GEMMs FOR PRE-/POST-/COCLINICAL ANALYSES}

The two key factors in the success of pre-/co-/postclinical trials in GEMMs are whether a model is "workable" for this type of analysis and how closely the model recapitulates the disease as it occurs in humans. These may seem obvious points, but in actuality the relevance of any given model needs to be addressed on a case-by-case basis, as there is no tried and true formula of applicability.

First, it is important to distinguish GEMMs from models based on implantation of primary tumors from the patients transplanted into recipient immunocompromised mice (e.g., patientderived xenograft or PDX models). It must be remembered, however, that a PDX approach is not always feasible, as some primary tumor types may not readily engraft in mice, as is the case in prostate for example. Importantly, GEMMs and PDX models can be regarded as complementary and not interchangeable for two main reasons: (i) PDX models are generated in immunocompromised mice, whereas GEMM models should be immune competent, and (ii) PDX models are genetically heterogeneous, whereas GEMMs are genetically identifiable by their engineered genetic alterations.

Among the important practical considerations in deciding whether a GEMM is "workable" for pre-/co-/postclinical analyses is the penetrance of the tumor phenotype and the latency to tumor growth. For example, if a model takes a very long time to form tumors ( $>1 \mathrm{yr})$, it may not be feasible to use in pre-/co-/postclinical trials; on the other hand, if a model has an exceedingly short survival time ( $\leq 2 \mathrm{mo}$ ), or acute mortality, it may also be impractical as the mice may die before the trial can be initiated or completed.

\section{BOX 2. THE MULTIPLE GOALS OF CLINICAL EFFORTS IN GEMMS}

1. Coclinical assessment of novel agents while in phase I and I/II, leading to the rapid stratification of responder and resistant populations

2. Assessment of single agents or combinations in GEMMs that exhibit genetically distinct and "rarer" cancer types/subsets (where sizable patient accrual represents a major hurdle)

3. Testing combinations in vivo to determine tolerability, efficacy, and pharmacodynamics

4. Facilitate prioritization of the most attractive combinations for assessment in humans

5. "Postclinical" optimization of SOC treatment modalities

6. Identifying mechanisms of de novo and acquired resistance to agents/therapeutic modalities (often even before clinical trials start)

7. Assessment of system impact of agents and combinations (e.g., tumor immune response or impact on tumor stroma and vasculature)

8. Coclinical and preclinical identification of novel biomarkers

9. Dissect the importance of temporal sequence of genetic alterations in dictating response or resistance to treatment modalities 
A second practical consideration is whether a GEMM is based on a conditional and/or inducible recombination, and therefore more likely to have a tissue-specific cancer phenotype, or whether it is based on a germline disruption, in which case the phenotype could be manifested in many tissues/cell types. In general, GEMMs with tissue-restricted phenotypes are more practical for pre-/co-/postclinical analyses as there are fewer confounding phenotypes. Moreover, many germline models of relevant tumor-suppressor alleles are embryonically lethal, reflecting their essential functions in the organism; as a consequence, their cancer phenotypes have to be studied in the heterozygous context. For example, germline loss of function of Pten results in embryonic lethality, and therefore analyses of the consequences of this condition must be performed in heterozygotes, which develop tumors in various tissues with differing latencies. Because of this constraint, analyses of conditional Pten alleles have proven to be much more feasible for pre-/co-/postclinical trials.

In addition to these practical considerations, an important consideration is whether a given model effectively recapitulates key pathways involved in the genesis of a given cancer. Or, simply put, whether the GEMM actually models its human counterpart. Notably, the relevance of early generation GEMMs, many of which were based on tissue-specific expression of SV40 oncoproteins, has often been called into question, as cancers in human tissues rarely arise as a consequence of SV40 oncogene expression. In the prostate, for example, most SV40-based transgenic models develop neuroendocrine tumors rather than adenocarcinoma; in the endocrine pancreas, however, the SV40-based RIP-TAG model developed by Hanahan (1984) has proved to be one of the most relevant and useful GEMMs in a variety of applications, particularly pre-/co-/postclinical investigations.

That said, it is more generally the case that GEMMs based on perturbations of genes and pathways that actually occur in the cancer under study are more relevant and suitable models for pre-/co-/ postclinical investigations, particularly because they can illuminate the consequences of targeting relevant pathways. For example, Kras mutations represent a major initiating event in both pancreatic and lung cancer, and GEMMs based on Kras activation in these tissues have proven to be of great value to pre-/co-/postclinical investigations (see Singh et al. 2012). Similarly, one of the key genetic alterations in melanoma is mutation of Braf and, accordingly, the most relevant GEMMs to the elucidation of melanoma biology are based on its activation (see, e.g., Dankort et al. 2009). On the other hand, although GEMMs may model relevant pathways associated with a given cancer, they may not precisely mimic the genetic alterations that occur. For example, most GEMMs with conditional loss of Pten in the prostate have deletion of both alleles; however, in human prostate cancer, Pten inactivation is much more complex, and rarely is it the case that both alleles are deleted in the absence of other mutations (see Shen and Abate-Shen 2010).

Another important consideration regarding the appropriateness of a given GEMM for pre-/co-/ postclinical analysis is the relevance of the cell type in which the gene recombination in question occurs. In some tumor types, the identity of the originating cells is a matter of considerable debate. However, for those systems in which this is established, the greatest limitation is the availability of appropriate Cre drivers, which are usually based on tissue-specific expression but may not have the ability to direct gene recombination to appropriate cells of origin. Some Cre drivers, however, such as the Nkx3.1 ${ }^{\text {CreERt2 }}$ allele, can accurately direct gene recombination to the appropriate cell of origin (in this case of prostate cancer) (Wang et al. 2009). Another approach is to use viral transduction, such as Adeno-Cre vectors, which can target recombination in multiple cell types (Jackson et al. 2001), or to use directed promoters that would restrict expression to selected cell types upon viral transduction, as has been done in the lung.

An additional limitation is that in GEMMs that are based on recombination of multiple genes, the genes in question are inactivated simultaneously, as they are generally driven by a common Cremediated event, which makes it difficult to model sequential events that may be more relevant to disease progression. However, the availability of FLP-mediated recombination may make it more feasible to achieve sequential recombination using independent CRE- and FLP-driven drivers (Young et al. 2011).

With all of these caveats, how is it possible to credential pre-/co-/postclinical studies in GEMMs for clinically relevant investigations? One major criterion for evaluating the appropriateness of a 
GEMM would be to determine whether the molecular pathways altered in the model are conserved in the corresponding human cancer. In other words, regardless of whether the initiating events are accurately modeled, the GEMMs may be considered appropriate if they share similar conserved molecular pathways with human patients that are relevant to disease initiation or progression (see, e.g., Ellwood-Yen et al. 2003; Sweet-Cordero et al. 2005). A second essential criterion for the appropriateness of a given GEMM would be the relevance of its histopathological phenotype, which should be characterized in detail.

In summary, selecting the appropriate GEMM for pre-/co-/postclinical investigations depends in part on practical issues that impact experimental feasibility and in part on the biological relevance of the model for appropriately mimicking the phenotype. However, the latter is also dependent on practical issues, including the availability of relevant targeting alleles and appropriate drivers for gene recombination. Thus, an "appropriate" model is one that accurately recapitulates the phenotype of disease progression and is also a sensible choice for experimentation.

\section{HOW TO DESIGN PRE-/POST-/COCLINICAL TRIALS}

With the appropriate GEMMs in hand, the next step is to design trials that are most relevant to ongoing or planned clinical trials. In general, a good strategy uses a team-based approach that incorporates the input of clinicians, mouse modelers, statisticians, pharmacologists, imagers, and pathologists, who together contribute to the design of the trial, in a manner similar to the typical team-based approach that is used to design most clinical trials. It is important to include all of the key players in the initial discussions to plan the trials, as this will ensure that the parameters of the trial are developed in an optimal way and that the end points are relevant, manageable, and informative. This is obviously of paramount importance if a drug is tested "coclinically."

The most straightforward starting points for these discussions are the choice of drugs to be tested, and whether the GEMM models the most relevant patient population. However, considerations of study design such as cohort size and appropriate end points are essential to ensure that useful information will be obtained. When designing a trial the following key points need to be taken into consideration.

- Relevance to ongoing clinical trials. First and foremost, an important consideration is whether a planned GEMM trial addresses a relevant clinical issue and evaluates clinically relevant drugs. For example, in prostate cancer, the major clinical direction is focused on agents that target androgen receptor signaling, and therefore "coclinical" perspective analyses of such agents would be extremely relevant moving forward.

- Statistical power. It is very important to design studies that have sufficient statistical power to make strong conclusions. GEMM trials with insufficiently sized cohorts are not likely to prove meaningful. Although this seems an obvious point, it is too often overlooked.

- Pharmacokinetics/dynamics. Whether and how a drug of interest is taken up into the tumor, and whether the drug is metabolized similarly in mice and humans, are important considerations in terms of the interpretation of relevant findings, and therefore need to be accurately measured to interpret the results of a GEMM trial. In particular, recent studies have implied that one of the ways tumors develop resistance to drugs is by blocking drug uptake, as influenced by the tumor microenvironment. This can be effectively evaluated in GEMMs.

- Imaging. Most trials in GEMMs will incorporate imaging to evaluate in real time the tumor response to the drug treatment being tested. Notably, the type of imaging modality that is appropriate for a given trial depends on the question being addressed. For example, magnetic resonance imaging (MRI) may be effective in elucidating tumor response, whereas positron emission tomography (PET) imaging is more relevant to evaluating the response of metastatic lesions. 
- Clinically relevant end points. Designing relevant trials with GEMMs requires the determination of clinically relevant end points. For example, suppression of tumor volume and cell proliferation as well as induction of apoptosis of tumor cells are important end points in considering whether a drug is effective. However, improved survival and decreased metastatic spread are likely to be more clinically relevant end points. Another important consideration is whether the trial should be done using GEMMs in which the tumors have been surgically removed before drug delivery, especially if this is what is currently done in humans, which, however, may be difficult to achieve in certain tumor types.

- Tissue collection, pathology, and bioinformatics. The ability to acquire serially and temporally distinct tumor specimens, or microdissected portions of a tumor lesion, in any given GEMM subjected to any given treatment modality, obviously represents one of the strengths offered by the use of GEMMs of cancer. However, this should not distract the researcher from always ensuring that model-derived specimens are compared with appropriate human specimens collected and processed in a similar manner and at comparable biological and temporal stages of the tumor's natural history. Similarly, it is of paramount importance that pathology, imaging, and bioinformatics analyses be integrated using universal platforms.

\section{THE FUTURE OF MODEL SYSTEMS IN DRUG TESTING}

The availability of numerous and faithful cancer GEMMs render it feasible to scale up their utilization in "mouse hospitals" to guide and improve clinical intervention in a variety of ways. In future years, we expect that modeling efforts will be further refined to incorporate new GEMMs for distinct types of genetic determinants of cancer. This will further expand the repertoire of relevant cancer GEMMs and eventually allow a comprehensive representation of the complexity of human cancer in the mouse hospital. These models will be invaluable tools in both rapid testing (singly and in combination) and the identification of appropriate indications of use in specific cancer subtypes for the panoply of novel targeted agents to be developed in the years to come.

\section{REFERENCES}

Dankort D, Curley DP, Cartlidge RA, Nelson B, Karnezis AN, Damsky WE Jr, You MJ, DePinho RA, McMahon M, Bosenberg M. 2009. $B r a f^{\mathrm{V} 600 \mathrm{E}}$ cooperates with Pten loss to induce metastatic melanoma. Nat Genet 41: 544-552.

Ellwood-Yen K, Graeber TG, Wongvipat J, Iruela-Arispe ML, Zhang J, Matusik R, Thomas GV, Sawyers CL. 2003. Myc-driven murine prostate cancer shares molecular features with human prostate tumors. Cancer Cell 4: 223-238.

Hanahan D. 1984. Tumour development: Oncogenes in transgenic mice. Nature 312: 503-504.

Jackson EL, Willis N, Mercer K, Bronson RT, Crowley D, Montoya R, Jacks T, Tuveson DA. 2001. Analysis of lung tumor initiation and progression using conditional expression of oncogenic K-ras. Genes Dev 15: 3243-3248.

Nardella C, Lunardi A, Patnaik A, Cantley LC, Pandolfi PP. 2011. The APL paradigm and the "co-clinical trial" project. Cancer Discov 1: 108-116.
Shen MM, Abate-Shen C. 2010. Molecular genetics of prostate cancer: New prospects for old challenges. Genes Dev 24: 1967-2000.

Singh M, Murriel CL, Johnson L. 2012. Genetically engineered mouse models: Closing the gap between preclinical data and trial outcomes. Cancer Res 72: 2695-2700.

Sweet-Cordero A, Mukherjee S, Subramanian A, You H, Roix JJ, LaddAcosta C, Mesirov J, Golub TR, Jacks T. 2005. An oncogenic KRAS2 expression signature identified by cross-species gene-expression analysis. Nat Genet 37: 48-55.

Wang X, Kruithof-de Julio M, Economides KD, Walker D, Yu H, Halili MV, Hu Y-P, Price SM, Abate-Shen C, Shen MM. 2009. A luminal epithelial stem cell that is a cell of origin for prostate cancer. Nature 461: 495-500.

Young NP, Crowley D, Jacks T. 2011. Uncoupling cancer mutations reveals critical timing of p53 loss in sarcomagenesis. Cancer Res 71: 4040-4047. 


\section{Effective Utilization and Appropriate Selection of Genetically Engineered Mouse Models for Translational Integration of Mouse and Human Trials}

Cory Abate-Shen and Pier Paolo Pandolfi

Cold Spring Harb Protoc; doi: 10.1101/pdb.top078774 originally published online September 30, 2013

\begin{tabular}{cc}
$\begin{array}{r}\text { Email Alerting } \\
\text { Service }\end{array}$ & Receive free email alerts when new articles cite this article - click here. \\
\hline $\begin{array}{c}\text { Subject } \\
\text { Categories }\end{array}$ & $\begin{array}{c}\text { Browse articles on similar topics from Cold Spring Harbor Protocols. } \\
\text { Transgenic Mice (139 articles) }\end{array}$ \\
\hline
\end{tabular}

